\title{
Digitalisierung in der Beratung
}

\author{
Beate Fietze $\cdot$ Heidi Möller
}

Online publiziert: 11 . Juli 2018

(C) Springer Fachmedien Wiesbaden GmbH, ein Teil von Springer Nature 2018

Die Auseinandersetzung um die technischen und sozialen Folgen der digitalen Revolution steht seit geraumer Zeit im Mittelpunkt gesellschaftlicher und politischer Diskurse. Auch wenn die Meinungen darüber auseinandergehen, wie stark diese bereits in die jeweiligen gesellschaftlichen Teilbereiche eingreift, besteht doch darüber Konsens, dass der Trend der Digitalisierung sich unaufhaltsam durchsetzen wird. In der Politik und im öffentlichen Sektor nicht weniger als in den Unternehmen werden daher gegenwärtig Programme aufgesetzt, um eine (Selbst-)Positionierung zu erarbeiten - gerade, weil die Umsetzung für viele im eigenen Kontext immer noch ,Neuland“ ist.

Das gilt ebenfalls für das Feld der personenbezogenen Beratung. Auch die Berater/innen befindet sich aktuell in einer Phase der Selbstklärung in ihrem Verhältnis zur Digitalisierung. Auf Kongressen und in Publikationen werden die Möglichkeiten und Konsequenzen der Digitalisierung für die Beratung gegenwärtig intensiv diskutiert. Die grundlegende Frage lautet: Wie können wir durch den digitalen Wandel hindurch dem professionellen Anspruch der Berater/innen gerecht werden und zugleich die eigenen Beratungsangebote auf dem Markt behaupten?

Abseits der Debatten ist die tatsächliche Nutzung von digitalen Medien im Coaching - zumindest im deutschsprachigen Raum - jedoch noch sehr gering (Middendorf 2016, Umfrage von 2015/2016). Offensichtlich bewegen sich die Berater/ innen im Umgang mit der Digitalisierung in unterschiedlichen Geschwindigkeiten. Nur eine sehr kleine Gruppe unter ihnen hat sich bereits intensiv auf die neuen Rah-

Dr. B. Fietze $(\bowtie)$

Düsseldorfer Str. 4, 10719 Berlin, Deutschland

E-Mail: beate.fietze@snafu.de 
menbedingungen ausgerichtet und die digitalen Medien in ihre Arbeitsweise mit ihren Klient/innen integriert. Die überwiegende Mehrheit der Kolleg/innen arbeitet weiterhin in Face-to-Face-Settings.

Diese Zurückhaltung - und auch Skepsis - ist wenig erstaunlich. Die personenbezogene Beratung steht in der Tradition des Dialogs in der persönlichen Begegnung zwischen Berater und Klient. „Technik“ erscheint den meisten im Rahmen ihrer beraterischen Praxis als „kulturfremd“. Die möglichen Vorzüge der neuen Medien für die Beratung rücken erst allmählich ins Bild. Dies kann sich jedoch bald ändern. Noch sind die älteren Berater/innen die größte Altersgruppe im Coaching (Middendorf 2016) - in dem Maße wie die jüngeren Kohorten der Digital Natives in dieses Berufsfeld nachrücken, wird sich jedoch auch das Verhältnis zur Mediennutzung in der Beratung wandeln. Für die Digital Natives ist die digitalisierte Medienumgebung ihre biographische Primärerfahrung. Es ist daher sehr wahrscheinlich, dass die jüngeren Kolleg/innen eine unbefangenere und versiertere Integration der Mediennutzung in der Beratung praktizieren werden.

Aus verschiedenen Perspektiven und vor dem Hintergrund unterschiedlicher Erfahrungen leisten alle hier vertretenen Autor/innen zur Klärung und Selbstpositionierung des Verhältnisses von Digitalisierung und Beratung einen instruktiven Beitrag.

Die Durchsetzung der Digitalisierung wird gleichermaßen von der Faszination an deren ungeahnten technischen Möglichkeiten wie von dem von ihr ausgehenden ökonomischen Zugzwang forciert. Angesichts dieses kollektiven Sogs der Digitalisierung suchen Beate Fietze und Thomas Bachmann im ersten Hauptbeitrag deren Einfluss auf die zukünftige Entwicklung im Coaching abzuschätzen. In ihrer Analyse der Potentiale und Risiken der neuen Medien für die wesentlichen Aspekte im Coaching gerät die „Plattformökonomie“ nicht zufällig ins Visier. Durch das Ineinandergreifen der technischen und der ökonomischen Logik nimmt die Macht des Marktes im Coaching weiter zu und wird zu einer ernstzunehmenden Herausforderung für den Ermessensspielraum professionellen Handels.

In Anbetracht der Omnipräsenz der Kommunikationstechnologien im Arbeitsalltag der Coachingklient/innen plädiert Katja Kantelberg für eine größere Offenheit gegenüber dem „E-Weg“ im Coaching - sei es in Form des reinen E-Coachings, des blended Coachings, das analoge und virtuelle Begegnungsformen kombiniert, oder des immersiven Coachings, das ein „Eintauchen“ in eine permanent verfügbare virtuelle 3D-Umgebnung erlaubt. Gerade gegenüber der dem E-Coaching häufig unterstellten Qualitätsminderung der Beratungsbeziehung stellt die Autorin neue Formen der Nähe heraus, die einen direkteren und beständigeren Kontakt zu den aktuellen Beratungs- und Reflexionsbedarfen der Klient/innen ermöglichen. Hier wird spürbar: Wir sind bereits mittendrin und doch noch am Anfang einer spanenden Entwicklung.

Karin Martens-Schmid vergleicht die sensorischen und kommunikativen Dimensionen des Face-to-Face-Settings mit den neuen virtuellen Begegnungsszenarien im Coaching: Sehen und Hören bleiben zwar auch im Online-Coaching relevant, aber sie werden voneinander entkoppelt und neu gewichtet. Die Entwicklung immer subtilerer sensorischer Simulationen des Ausgangsmodells und deren Einfluss auf die Wahrnehmungen und das Ausdrucksverhalten im Coaching wird fortschreiten, und zwar so weitgehend, dass die Autorin die Frage stellt, ob das Präsenz-Setting auch 
künftig als Maßstab für die virtuellen Dialogräume gelten wird. Schon jetzt steht zu erwarten, dass diese Entwicklungen die Bedeutung des Face-to-Face-Settings verändern werden.

Jessica Huss und Christiane Eichenberg führen die Leser in die virtuellen Welten der Serious Games ein, einer besonderen Form der Onlinespiele, die sich als computerbasierte Lernwelten nutzen lassen. Mit Serious Games gelingt Lernen auf spielerische Weise. Lerntheorien haben längst gezeigt, dass der Erlebenszustand des Flows im Spiel eine ideale Wirkungsbedingung darstellt. Dies gilt vor allen für das Erlernen von Verhaltensänderungen, wie sie im Coaching häufig angestrebt werden. Die Abwägung der Autorinnen von Nutzungsrisiken und Anwendungsvorteilen zeigen aber auch, dass Serious Games nicht einfach ins Coaching übernommen werden können. Deren Weiterentwicklung muss sich an den Bedürfnissen der Coachingbranche ausrichten. Damit das gelingen kann, bedarf es eines Austausches zwischen den Plattform- und Spieleentwickler/innen und den Coaches.

In einem weiteren Hauptbeitrag zeigt Antje Schmitt den Stand der Forschung auf, wie die Herausforderung Ruhestand erfolgreich bewältigt werden kann. Diese Faktoren sind nicht nur höchst relevant für die Schar z. B. der Supervisor/innen, die doch recht vorangeschritten Alters sind - im Sinne der Selbstanwendung. Auch in der Beratung der Kund/innen ist der Aspekt Begleitung out of the job als Teil der Personalentwicklung ein wichtiges Segment. Hier lassen sich viele Anregungen zum Themenkreis Vorbereitung auf den Ruhestand entnehmen.

Es folgen drei Praxisberichte zum Themenschwerpunkt: Im Interview mit dem Coach Andreas Knierim beschreibt Claudia Bredt ihre Erfahrungen mit einem Telefoncoaching. Ihren Berater hat sie nie gesehen - über ein Jahr haben beide nur den auditiven Sinneskanal genutzt. Der Beitrag verhilft uns zu einer reflektierten Innenschau neuer Coachingformate aus Kund/innenperspektive. Und wer kann diese differenzierter darstellen als eine Person, die selbst seit Jahren analog erfolgreich als Supervisorin, Coach und Organisationsberaterin tätig ist?

Andreas Knierim selbst hat seine Coachingpraxis komplett digitalisiert. Kein Schnipsel Papier lässt sich in seinen Arbeitsräumen auffinden! Er lotet die Möglichkeiten des digitalen Beratungsalltags aus und hinterlässt mit seiner radikaleren Umstellung - neben der Bewunderung - sicherlich nicht nur bei mir den Eindruck: „Meine Güte, wie altmodisch bin doch ich in meiner Berufspraxis!“.

Katrin Oellerich illustriert ein interkulturelles Webinar zu sozialpsychologischen Themen in einer Integrationsmaßnahme für ausländische Wissenschaftler/innen. Dieser Ansatz ist nicht nur für Trainer/innen und Berater/innen interessant. Es lassen sich ebenso sinnvolle Felder erahnen, dieses Format in der Weiterbildung (nicht nur von Supervisor/innen, Coaches und Organisationsberater/innen) zu implementieren, Personalentwicklung zu innovieren und in Komplementärberatungssettings zu kreieren, in denen es neben den selbstreflexiven Elementen auch um Lerninhalte geht.

Als Innovation in der OSC startet in diesem Heft ein konzeptueller Disput. In jedem Heft wird es eine pointierte Rede zu Beratungskonzeptionen geben, und die Gegenrede erfolgt im nächsten Heft. Siegfried Greif verantwortet diese Rubrik und startet die Reihe mit einer kritischen Analyse des NLP. Weitere Kontroversen sind zu den Themen: Konstruktivismus, Psychodynamische Beratung, Systemische Ansätze, 
Neurowissenschaftliche Orientierung in der Beratung, Positive Psychologie, u.v. m. geplant. Auch hier sind Leser und Leserinnen herzlich eingeladen, Vorschläge zu machen und/oder eigene Beiträge einzureichen.

Im letzten Jahr starteten wir die Reihe „Filmanalysen“. In der letzten Begegnung der Herausgeber/innen und der Verlagsvertreterinnen mit dem wissenschaftlichen Beirat beschlossen wir, die Rubrik in „Kulturanalysen“ umzutaufen. Auf diese Weise wollen wir auch anderen künstlerischen Resonanzkörpern wie Literatur, Theater und Kunstwerken Raum geben, die auf wichtige gesellschaftliche Entwicklungen hinweisen. Analyse und Beschreibungen von Kulturgütern, die als Hintergrund für Beratung von Bedeutung sind, können an dieser Stelle ihren Platz finden. Auch an dieser Stelle sind Sie, liebe Leserinnen und Leser herzlich eingeladen, sich aktiv zu beteiligen.

Die Filmanalyse dieses Heftes „Das Leben der Anderen“, der Oskar gekürte Film von Florian Henckel von Donnersmarck, macht das Spannungsfeld zwischen Staatssicherheit und der Welt der Künstlerinnen und Künstler Mitte der 80er Jahre in der DDR auf. Er zeigt den Verfall einer Organisation des Misstrauens und des Verdachts inmitten der politischen und kulturellen Veränderungsprozesse und die Involvierung der handelnden Subjekte. Damit verweist auch er auf eine mögliche Schattenseite der Digitalisierung, dem Schwerpunktthema dieses Heftes.

\section{Literatur}

Middendorf, J. (2016). Coaching-Umfrage 2015/2016. https://coachingumfrage.files.wordpress.com/2017/ 04/ergeb-coaching-umfrage-2015.pdf. Zugegriffen: 20. Juni 2018. 